\title{
Spin and momentum of the light fields in inhomogeneous dispersive media with application to surface plasmon-polariton waves
}

\author{
${ }^{1,2}$ Bekshaev A.Y. and ${ }^{2,3}$ Bliokh K.Y. \\ ${ }^{1}$ I. I. Mechnikov National University, Research Institute of Physics, Dvorianska 2, \\ 65082, Odesa, Ukraine, e-mail: bekshaev@onu.edu.ua \\ ${ }^{2}$ Center for Emergent Matter Science, RIKEN, Wako-shi, Saitama 351-0198, Japan \\ ${ }^{3}$ Nonlinear Physics Centre, RSPE, The Australian National University, Canberra, \\ Australia, e-mail:k.bliokh@gmail.com
}

Received: 09.01 .2018

\begin{abstract}
Following the recent approach [Phys. Rev. Lett. 119, 073901 (2017); New J. Phys., 19, 123014 (2017)], we refine and accomplish a general scheme for the unified description of momentum and angular momentum of the light fields in complex material media. Equations for the canonical (orbital) and spin linear momenta, as well as the orbital and spin angular momenta are presented for a lossless inhomogeneous dispersive medium in a compact form, which is analogous to the Brillouin relationship for the energy. The results are applied to a surface plasmon-polariton field. The microscopic calculations support the phenomenological expectations. Our refined general scheme describes correctly the known unusual properties of the surface plasmon-polariton associated with transverse spin and magnetization momentum. Moreover, it predicts a singular momentum contribution sharply localized at the metal-dielectric interface, which is confirmed by the microscopic analysis. Our results can be useful for the optical systems employing structured light, especially in microoptics, plasmophotonics, optical sorting and micromanipulation.
\end{abstract}

Keywords: electromagnetic momentum, angular momentum, dispersive media, spin-orbital decomposition, Abraham-Minkowski dilemma, surface plasmonpolariton

PACS: 42.25.Bs, 42.50.Tx, 42.50.Nn, 42.50.Wk, 03.50.De

UDC: $535,537.8,621.371$

\section{Introduction}

Properties of structured light fields attract great attention during the past decade [1-3]. Such fields are necessary elements of various modern applications aimed at optical trapping, sorting, delivering, selective treatment, positioning and some other precise manipulations with extremely small quantities of matter [4-7]. In these areas, the dynamical characteristics of optical fields, first of all the spatial distribution of their energy, as well as their momentum and angular momentum (AM), play a crucial role. Therefore their investigations are highly relevant. It is very important that electromagnetic interactions in such systems usually develop against the background of highly inhomogeneous materials, which invokes the problem of 'structured light in structured media'.

Unfortunately, up to the recent time, the very instruments of description of the dynamical characteristics have been well established only for the case when the field evolves in free space. Even the introductory definitions of the field momentum become controversial and ambiguous in the case if continuous material media are present. A debate between the momentum paradigms 
originated from Abraham and Minkowski continues over a hundred years [8-11]. The most reasonable 'resolutions' of this dilemma find the arguments that support each of the sides and treat the both momenta as different physical quantities, with their own scopes and abilities. However, the analyses known up to date have been mostly limited to homogeneous media and plane-wavelike fields. Additional difficulties arise if a medium reveals any dispersion, i.e. the material parameters (the permittivity and/or the permeability) depend upon the light frequency. The only field characteristic for which the dispersion can be taken into account in a regular and consistent way is the energy whose density can be described by a famous Brillouin formula [12, 13].

Recently, Philbin and Allanson [14, 15] have made an important advance in the field by suggesting a regular and consistent way to describe the momentum and the AM in the dispersive media. Nonetheless, a genuine value of their approach becomes clear only in conjunction with a so-called canonical decomposition of the field momentum, when it is subdivided into the spin and canonical (orbital) components [16, 17]. With further elaboration and microscopic substantiation, this approach has resulted in unified, compact and physically transparent expressions for the canonical linear momentum, as well as the orbital and spin AMs of optical fields in lossless, inhomogeneous dispersive media. The methods and results of Refs. [16, 17] have enabled elaborating a rigorous, consistent theory of surface plasmon-polaritons (SPPs), analyzing thoroughly their non-trivial properties (e.g., a transverse spin) and predicting a number of novel phenomena (e.g., an SPP-induced magnetization of media).

The present work is aimed to further refine the methodology reported in Refs. [16, 17]. In particular, we enhance their general scheme to include a linear spin momentum whose description in the dispersive media has earlier been omitted. As we will see, this enables one to obtain a full set of instruments for the description and analysis of electromagnetic momentum and AM in the dispersive media, and to shed new light on some important results of the earlier works associated with the SPP properties. In particular, we reveal some peculiar features of the momentum and spin distributions associated with near-surface contributions and physically essential singular components of the momentum sharply localized at the metal-dielectric interface. The present consideration is essentially based on the materials of Refs. [16, 17]; we not only employ their main ideas but, where possible, adhere to their notation and terminology.

\section{General overview of the dispersion-modified description of optical momentum in media}

Below we deal with monochromatic fields in lossless dielectric media where the electric and magnetic vectors $\mathcal{E}(\mathbf{r}, t)=\operatorname{Re}\left[\mathbf{E}(\mathbf{r}) e^{-i \omega t}\right]$ and $\mathcal{H}(\mathbf{r}, t)=\operatorname{Re}\left[\mathbf{H}(\mathbf{r}) e^{-i \omega t}\right]$ obey the Maxwell equations,

$$
\nabla \cdot(\mu \mathbf{H})=0, \quad \mu \mathbf{H}=-\frac{i}{k_{0}} \nabla \times \mathbf{E}, \quad \nabla \cdot(\varepsilon \mathbf{E})=0, \quad \varepsilon \mathbf{E}=\frac{i}{k_{0}} \nabla \times \mathbf{H} .
$$

Here the medium is characterized by the real permittivity $\varepsilon=\varepsilon(\omega, \mathbf{r})$ and permeability $\mu=\mu(\omega, \mathbf{r})$ that can depend on the coordinates (inhomogeneity) and the frequency (dispersion), $k_{0}=\omega / c$ is the free-space wave number, and $c$ the vacuum light velocity. The only dynamical property of the field whose definition is well established and free from controversies in such conditions is the electromagnetic energy, with its density described by the well-known Brillouin expression $[12,13]$ : 


$$
\begin{aligned}
& \tilde{W}=\frac{g \omega}{2}\left(\tilde{\varepsilon}|\mathbf{E}|^{2}+\tilde{\mu}|\mathbf{H}|^{2}\right), \\
& \tilde{\varepsilon}=\varepsilon+\omega \frac{d \varepsilon}{d \omega}, \quad \tilde{\mu}=\mu+\omega \frac{d \mu}{d \omega},
\end{aligned}
$$

where $g=(8 \pi \omega)^{-1}$ [From now on, all dispersion-modified electromagnetic quantities will be marked by tildes " $\sim$ "]. Note a neat and unified form of this expression, which is also valid for the inhomogeneous media and differs from the dispersion-free formula [12, 13] just by replacement $(\varepsilon, \mu) \rightarrow(\tilde{\varepsilon}, \tilde{\mu})$. Regrettably, there is no such a straight way if one tries to generalize the field momentum and the AM $[16,17]$.

For the case of negligible dispersion, the Abraham and Minkowski momentum densities are given by [8-12]

$$
\begin{aligned}
& \mathcal{P}_{A}=g k_{0} \operatorname{Re}\left(\mathbf{E}^{*} \times \mathbf{H}\right), \\
& \mathcal{P}_{M}=g k_{0} \varepsilon \mu \operatorname{Re}\left(\mathbf{E}^{*} \times \mathbf{H}\right) .
\end{aligned}
$$

These momenta are sometimes referred to as 'kinetic', because they appear in the kinetic (symmetrical) energy-momentum tensor [18] of the electromagnetic field. Abstracting from the Abraham-Minkowski dilemma [8-11], the both kinetic momenta encounter difficulties when being applied to the structured light fields $[16,17]$. Besides, the corresponding AM densities

$$
\mathcal{J}_{A, M}=\mathbf{r} \times \mathcal{P}_{A, M}
$$

are 'extrinsic' (i.e., depend on the choice of coordinate origin), and the kinetic formalism based on Eqs. (4) or (5) cannot describe separate contributions of spatial ('orbital') and polarization ('spin') degrees of freedom of light. At the same time, the latter represent important subjects of modern optics, which are extensively studied [3, 19]. These drawbacks are partly eliminated in the 'canonical' approach associated with the spin-orbital decomposition of the field AM [16-18, 2022]. The relevant procedure manifests especially favourable properties of the Minkowski momentum (5), which can be represented as

$$
\mathcal{P}_{M}=\mathbf{P}_{M}+\mathbf{P}_{M}^{S}, \quad \mathbf{P}_{M}^{S}=\frac{1}{2} \nabla \times \mathbf{S}_{M},
$$

where

$$
\mathbf{P}_{M}=\frac{g}{2} \operatorname{Im}\left[\varepsilon \mathbf{E}^{*} \cdot(\nabla) \mathbf{E}+\mu \mathbf{H}^{*} \cdot(\nabla) \mathbf{H}\right]
$$

denotes the 'canonical' momentum, and

$$
\mathbf{S}_{M}=\frac{g}{2} \operatorname{Im}\left(\varepsilon \mathbf{E}^{*} \times \mathbf{E}+\mu \mathbf{H}^{*} \times \mathbf{H}\right)
$$

is the Minkowski spin density. The representation given by Eqs. (7)-(9) is grounded on the Maxwell equations (1) and, remarkably, it holds for arbitrary spatially dependent $\varepsilon$ and $\mu$ [Note that the similar operation with the Abraham momentum (4) is impossible because additional terms appear owing to medium inhomogeneity $[16,17]]$. Accordingly, the Minkowski AM (6) can be reduced to

$$
\mathcal{J}_{M}=\mathbf{r} \times \mathcal{P}_{M}=\mathbf{L}_{M}+\mathbf{S}_{M}
$$

Here

$$
\mathbf{L}_{M}=\mathbf{r} \times \mathbf{P}_{M}
$$

is the orbital $\mathrm{AM}$ representing extrinsic part of the total field $\mathrm{AM}$, for which $\mathbf{S}_{M}$ (9) is intrinsic part. Equation (10) is based on the non-local integral equality 


$$
\frac{1}{2} \int \mathbf{r} \times\left(\nabla \times \mathbf{S}_{M}\right) d V=\int \mathbf{S}_{M} d V=\int \mathbf{r} \times \mathbf{P}_{M}^{S} d V,
$$

which is valid for any fields that vanish properly at infinity. Eq. (12) expresses the following general rule: for any electromagnetic field with inhomogeneous spin density $\mathbf{S}$, the corresponding linear spin momentum $\mathbf{P}^{S}$ exists, with its density given by

$$
\mathbf{P}^{S}=\frac{1}{2} \nabla \times \mathbf{S} .
$$

The second relationship in Eq. (7) is a special case of this rule.

Note that, via the Noether theorem, the canonical momentum given by Eq. (8) follows directly from the field Lagrangian as a conserved quantity [18, 23, 24]; this derivation leads to the non-symmetric (canonical) energy-momentum tensor. Then the linear spin momentum $\mathbf{P}^{S}$ appears as an auxiliary means for tensor symmetrization by adding a solenoidal momentum component [25]. However, the recent studies (e.g., those reported in Refs. [16, 17, 20-22, 26, 27]), as well as Eqs. (7)-(9) and (13), disclose its deep physical meaning.

So far, the dispersion has been neglected in our reasoning. An important step to include the medium dispersion into the field momentum theory has been made in Refs. [14, 15], where the consideration is based on the field Lagrangian in a dispersive medium, and the momentum and AM are derived via the Noether theorem. This mode of operation has naturally led to the Minkowski-based momentum representation and resulted in the following expressions for the field momentum and the AM in the dispersive medium:

$$
\begin{aligned}
& \tilde{\mathcal{P}}_{M}^{P}=\mathcal{P}_{M}+\frac{g \omega}{2} \operatorname{Im}\left[\frac{d \varepsilon}{d \omega} \mathbf{E}^{*} \cdot(\nabla) \mathbf{E}+\frac{d \mu}{d \omega} \mathbf{H}^{*} \cdot(\nabla) \mathbf{H}\right], \\
& \tilde{\mathcal{J}}_{M}=\mathbf{r} \times \tilde{\mathcal{P}}_{M}+\frac{g \omega}{2} \operatorname{Im}\left[\frac{d \varepsilon}{d \omega} \mathbf{E}^{*} \times \mathbf{E}+\frac{d \mu}{d \omega} \mathbf{H}^{*} \times \mathbf{H}\right]
\end{aligned}
$$

(the superscript in Eq. (14) implies that the dispersion correction has been performed by means of the Philbin's procedure). However, the authors of Refs. [14, 15] have not employed the spinorbital decomposition (see Eqs. (7)-(10)), without which the real meaning of their approach is underestimated. Indeed, with allowance for Eqs. (8), (9) and (11), the results (14) and (15) can be presented as

$$
\tilde{\mathcal{P}}_{M}^{P}=\tilde{\mathbf{P}}_{M}+\mathbf{P}_{M}^{S}
$$

where

$$
\tilde{\mathbf{P}}_{M}=\frac{g}{2} \operatorname{Im}\left[\tilde{\varepsilon} \mathbf{E}^{*} \cdot(\nabla) \mathbf{E}+\tilde{\mu} \mathbf{H}^{*} \cdot(\nabla) \mathbf{H}\right]
$$

and

$$
\tilde{\mathcal{J}}_{M}=\tilde{\mathbf{L}}_{M}+\tilde{\mathbf{S}}_{M}
$$

with

$$
\tilde{\mathbf{S}}_{M}=\frac{g}{2} \operatorname{Im}\left(\tilde{\varepsilon} \mathbf{E}^{*} \times \mathbf{E}+\tilde{\mu} \mathbf{H}^{*} \times \mathbf{H}\right), \tilde{\mathbf{L}}_{M}=\mathbf{r} \times \tilde{\mathbf{P}}_{M} .
$$

Thus, the Philbin's transformations (14) and (15) provide explicit expressions for the densities of canonical momentum $\left(\tilde{\mathbf{P}}_{M}\right)$ and $\operatorname{spin} \operatorname{AM}\left(\tilde{\mathbf{S}}_{M}\right)$ of the optical field in the inhomogeneous dispersive medium. Note a remarkably compact and unified character of expressions (17) and (19): they merely reproduce a scheme in which the dispersion has been taken 
into account in the Brillouin formula (2) for the energy. In this form, equations for the field momentum and the AM have been derived and used for the analysis of the SPP [16, 17].

However, there are some imperfections in the pattern described by Eqs. (16)-(19):

(i) In Eq. (16), the spin momentum $\mathbf{P}_{M}^{S}$ 'does not feel' the dispersion and preserves the dispersion-free form given by Eqs. (7) and (9);

(ii) According to Eq. (13), the first relationship in Eq. (19) should entail the spin momentum expression which differs from that accepted in Eq. (16), i.e.

$$
\tilde{\mathbf{P}}_{M}^{S}=\frac{1}{2} \nabla \times \tilde{\mathbf{S}}_{M}=\frac{g}{4} \nabla \times \operatorname{Im}\left(\tilde{\varepsilon} \mathbf{E}^{*} \times \mathbf{E}+\tilde{\mu} \mathbf{H}^{*} \times \mathbf{H}\right) .
$$

Obviously, this discrepancy appears because the procedure of Ref. [14] based on the Noether theorem gives dispersion corrections to the conserved canonical momentum, whereas the divergence-free spin momentum should be considered separately. The corresponding independent result for the spin momentum is just provided by Eq. (20), i.e. one can correct Eq. (16) as

$$
\tilde{\mathcal{P}}_{M}=\tilde{\mathbf{P}}_{M}+\tilde{\mathbf{P}}_{M}^{S},
$$

which is equivalent to the following modification of the Philbin's relation (14):

$$
\begin{aligned}
\tilde{\mathcal{P}}_{M}= & \underbrace{}_{\tilde{\mathcal{P}}_{M}+\frac{g \omega}{2} \operatorname{Im}\left[\frac{d \varepsilon}{d \omega} \mathbf{E}^{*} \cdot(\nabla) \mathbf{E}+\frac{d \mu}{d \omega} \mathbf{H}^{*} \cdot(\nabla) \mathbf{H}\right]} \\
& +\frac{g \omega}{4} \operatorname{Im}\left[\nabla \times\left(\frac{d \varepsilon}{d \omega} \mathbf{E}^{*} \times \mathbf{E}+\frac{d \mu}{d \omega} \mathbf{H}^{*} \times \mathbf{H}\right)\right] .
\end{aligned}
$$

Finally, the system of Eqs. (16a), (20) and (17)-(19) completes description of the field momentum and the $\mathrm{AM}$ in the inhomogeneous dispersive media. It is the main general statement of this work; some of its consequences will be considered below.

\section{Application to surface plasmon-polariton}

Following Refs. [16, 17], we apply the equations derived above to a very representative and nontrivial example of structured optical fields in dispersive structured matter. It is the SPP wave at the metal-vacuum interface [4]. As seen from Fig. 1a, we consider an interface ( $x=0$ plane) that separates half-spaces of a vacuum $(x>0$; medium 1$)$ and a metal $(x<0$; medium 2), thus making the system inhomogeneous. The SPP wave is a highly structured double-evanescent wave that decays exponentially on the both sides of the interface and propagates along $z$ axis with a well-defined wave vector, $\mathbf{k}_{p}=k_{p} \overline{\mathbf{z}}$ [Hereafter, $\overline{\mathbf{x}}, \overline{\mathbf{y}}$ and $\overline{\mathbf{z}}$ denote the unit vectors of the corresponding axes]. The permittivity and permeability of metal are described by a standard plasma model [4],

$$
\mu=1, \quad \varepsilon(\omega)=1-\omega_{p}^{2} / \omega^{2}
$$

Here

$$
\omega_{p}^{2}=4 \pi n_{0} e^{2} / m
$$

is the plasma frequency, $n_{0}$ the volume density of free electrons in the metal, $e<0$ the electron charge, and $m$ the electron mass. Thus, the metal represents a dispersive medium with $\tilde{\varepsilon}=1+\omega_{p}^{2} / \omega^{2}=2-\varepsilon \neq \varepsilon$, and the dispersion is crucial for the SPP properties. Even the existence of the SPP is conditioned by the frequency limit $\omega<\omega_{p} / \sqrt{2}$, i.e. we have $\varepsilon<-1$ [4]. 

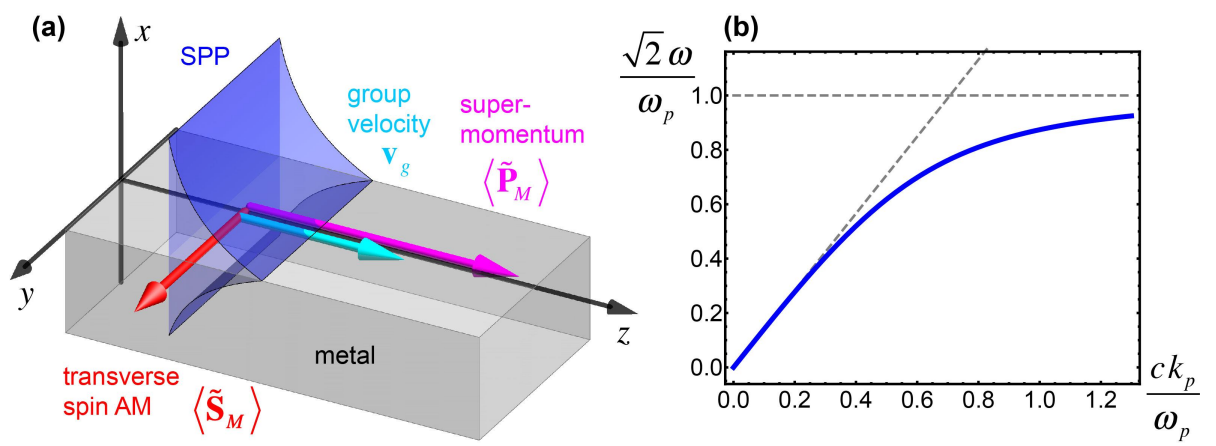

Fig. 1. (a) Scheme of SPP wave occurring at a metal-vacuum interface [4, 17]. A subluminal group velocity, super-momentum (see Ref. [17]) and a transverse spin (see Eq. (27)) are depicted; (b) Dispersion dependence $\omega\left(k_{p}\right)$ for the SPP as obtained from Eqs. (21) and (25).

The electric and magnetic fields of the SPP wave are described by the relations $[4,16,17]$

$$
\begin{aligned}
& \mathbf{E}=A\left\{\begin{array}{l}
\left(\overline{\mathbf{x}}-i \frac{\kappa_{1}}{k_{p}} \overline{\mathbf{z}}\right) \exp \left(i k_{p} z-\kappa_{1} x\right), \quad x>0 \\
\frac{1}{\varepsilon}\left(\overline{\mathbf{x}}+i \frac{\kappa_{2}}{k_{p}} \overline{\mathbf{z}}\right) \exp \left(i k_{p} z+\kappa_{2} x\right), \quad x<0
\end{array}\right. \\
& \mathbf{H}=A \begin{cases}\overline{\mathbf{y}} \frac{k_{0}}{k_{p}} \exp \left(i k_{p} z-\kappa_{1} x\right), & x>0 \\
\overline{\mathbf{y}} \frac{k_{0}}{k_{p}} \exp \left(i k_{p} z+\kappa_{2} x\right), & x<0,\end{cases}
\end{aligned}
$$

where $A$ denotes the field amplitude. The propagation constant $k_{p}$ and the spatial-decay constants $\kappa_{1}$ and $\kappa_{2}$ of the SPP field are as follows:

$$
k_{p}=\frac{\sqrt{-\varepsilon}}{\sqrt{-1-\varepsilon}} k_{0}, \quad \kappa_{1}=\frac{1}{\sqrt{-1-\varepsilon}} k_{0}, \quad \kappa_{2}=\frac{-\varepsilon}{\sqrt{-1-\varepsilon}} k_{0} .
$$

The dispersion curve for the SPP obtained from Eqs. (21) and (25) is shown in Fig. $1 b$.

After substituting Eqs. (23) and (24) into Eqs. (17) and (19), with using Eqs. (21) and (25), we obtain readily the canonical momentum distribution

$$
\tilde{\mathbf{P}}_{M}=g|A|^{2} \frac{k_{0}^{2}}{k_{p}} \overline{\mathbf{z}}\left\{\begin{array}{cl}
\frac{\varepsilon}{1+\varepsilon} \exp \left(-2 \kappa_{1} x\right), & x>0 \\
\frac{1-\varepsilon+\varepsilon^{2}}{\varepsilon(1+\varepsilon)} \exp \left(2 \kappa_{2} x\right), & x<0
\end{array}\right.
$$

(which matches Eqs. (3.4) and (3.9) of Ref. [17]), as well as the spin density of the SPP wave

$$
\tilde{\mathbf{S}}_{M}=g|A|^{2} \overline{\mathbf{y}}\left\{\begin{array}{cl}
\frac{\kappa_{1}}{k_{p}} \exp \left(-2 \kappa_{1} x\right), & x>0 \\
-\frac{\kappa_{2}}{k_{p}} \frac{2-\varepsilon}{\varepsilon^{2}} \exp \left(2 \kappa_{2} x\right), & x<0
\end{array}\right.
$$

(which corresponds to Eq. (3.13) of Ref. [17]). This spin AM has the opposite directions in the vacuum and metal; so, we have $S_{y}<0$ for $x<0$, which agrees with the opposite rotation 
directions of the electric field $\mathbf{E}$ in the contacting media (see Eq. (23)). Accordingly, the spin density (27) experiences a 'jump' at $x=0$ :

$$
\Delta \tilde{\mathbf{S}}_{M}=\tilde{\mathbf{S}}_{M}(x>0)-\tilde{\mathbf{S}}_{M}(x<0)=g|A|^{2} \frac{\kappa_{1}}{k_{p}} \frac{2(\varepsilon-1)}{\varepsilon} \overline{\mathbf{y}}=2 g|A|^{2} \frac{1-\varepsilon}{(-\varepsilon)^{3 / 2}} \overline{\mathbf{y}} .
$$

This is in contrast to the 'naïve' Minkowski spin (9) which is continuous at the interface:

$$
\mathbf{S}_{M}=g|A|^{2} \overline{\mathbf{y}} \begin{cases}\frac{\kappa_{1}}{k_{p}} \exp \left(-2 \kappa_{1} x\right), & x>0 \\ -\frac{\kappa_{2}}{k_{p} \varepsilon} \exp \left(2 \kappa_{2} x\right), & x<0\end{cases}
$$

Formula (27) provides an adequate description of the transverse spin of the SPP predicted earlier [24] and characterizes correctly the total spin of the SPP, which is proportional to $\int_{-\infty}^{\infty} \tilde{\mathbf{S}}_{M}(x) d x$ [17]. Now we make use of Eq. (27) for evaluating the linear spin momentum. To this end, one can notice that all types of the spin in the SPP geometry under consideration (see Fig. 1a) are $y$-directed and $z$-independent. Then Eq. (13) can be simplified to

$$
\nabla \times\left(\overline{\mathbf{y}} S_{y}\right)=\overline{\mathbf{z}} \frac{\partial S_{y}}{\partial x}
$$

Applying this to Eq. (27), one obtains

$$
\tilde{\mathbf{P}}_{M}^{S}=g|A|^{2} \frac{1-\varepsilon}{(-\varepsilon)^{3 / 2}} \overline{\mathbf{z}} \delta(x)+g|A|^{2} \frac{k_{0}^{2}}{k_{p}} \overline{\mathbf{z}} \begin{cases}\frac{1}{1+\varepsilon} \exp \left(-2 \kappa_{1} x\right), & x>0 \\ \frac{2-\varepsilon}{1+\varepsilon} \exp \left(2 \kappa_{2} x\right), & x<0 .\end{cases}
$$

It is helpful to compare this result with the 'dispersion-free' spin momentum $\mathbf{P}_{M}^{S}$ that follows from Eqs. (13), (30) and (7),

$$
\mathbf{P}_{M}^{S}=\frac{1}{2} \nabla \times \mathbf{S}_{M}=g|A|^{2} \frac{k_{0}^{2}}{k_{p}} \overline{\mathbf{z}}\left\{\begin{array}{ll}
\frac{1}{1+\varepsilon} \exp \left(-2 \kappa_{1} x\right), & x>0 \\
\frac{\varepsilon}{1+\varepsilon} \exp \left(2 \kappa_{2} x\right), & x<0
\end{array} .\right.
$$

The difference between Eqs. (31) and (32) exists only in the metal and at the interface $(x \leq 0)$. It can be written as

$$
\Delta \mathcal{P}=\frac{1}{2} \nabla \times\left(\tilde{\mathbf{S}}_{M}-\mathbf{S}_{M}\right)=\tilde{\mathbf{P}}_{M}^{S}-\mathbf{P}_{M}^{S}=(\Delta \mathcal{P})^{\text {surf }}+(\Delta \mathcal{P})^{\mathrm{vol}}
$$

with explicitly separated surface (singular) and volume contributions

$$
(\Delta \mathcal{P})^{\text {surf }}=g|A|^{2} \frac{1-\varepsilon}{(-\varepsilon)^{3 / 2}} \overline{\mathbf{z}} \delta(x), \quad(\Delta \mathcal{P})^{\mathrm{vol}}=-g|A|^{2} \frac{k_{0}^{2}}{k_{p}} \overline{\mathbf{z}} \frac{2(1-\varepsilon)}{-1-\varepsilon} e^{2 \kappa_{2} x}, x<0 .
$$

The term involving the delta function appears due to discontinuity of the spin AM (see Eqs. (27) and (28)), whereas the volume part of Eq. (34) describes the additional momentum contribution which has been 'lost' in the phenomenological SPP analysis in Section 3 of Ref. [17]. (Nonetheless, this contribution was implicitly 'found' in the subsequent sections of Ref. [17] presenting the microscopic approach, and we will demonstrate this in the next Section of this paper).

Now we briefly discuss some aspects of our new results expressed by Eqs. (31), (33) and (34). First of all, with allowance for Eqs. (25), the delta-function term in Eq. (34) ensures a zero 
value of the 'total' additional momentum (33) for the SPP cross-section,

$$
\langle\Delta \mathcal{P}\rangle=\int_{-\infty}^{\infty} \Delta \mathcal{P} d x=0
$$

as well as for the integral spin momentum,

$$
\left\langle\tilde{\mathbf{P}}_{M}^{S}\right\rangle=\int_{-\infty}^{\infty} \tilde{\mathbf{P}}_{M}^{S} d x=0
$$

This is what is required by the general theory [22, 23, 26], being associated with a divergence-less nature of the quantities given by Eqs. (20) and (33). Second, the correction (33) of the spin momentum (as we pass from Eq. (32) to Eq. (31)) is equivalent to transition from Eq. (14) to Eq. (14a), i.e. to adding the second line of Eq. (14a) to the Philbin's kinetic Minkowski momentum (14). Therefore, the 'true' kinetic momentum of the SPP is expressed by the relation

$$
\tilde{\mathcal{P}}_{M}=\tilde{\mathbf{P}}_{M}+\tilde{\mathbf{P}}_{M}^{S}=\tilde{\mathcal{P}}_{M}^{P}+\Delta \mathcal{P}=(\Delta \mathcal{P})^{\text {surf }}+\tilde{\mathcal{P}}_{M}^{\text {vol }}
$$

(see Eqs. (33) and (34)). It appears to be singular, due to the first relationship in Eq. (34). In Eq. (36), $\tilde{\mathcal{P}}_{M}^{P}$ corresponds to the Philbin's dispersive-medium momentum (14),

$$
\tilde{\mathcal{P}}_{M}^{P}=g|A|^{2} \frac{k_{0}^{2}}{k_{p}} \overline{\mathbf{z}}\left\{\begin{array}{c}
\exp \left(-2 \kappa_{1} x\right), \quad x>0 \\
\frac{1-\varepsilon+2 \varepsilon^{2}}{\varepsilon(1+\varepsilon)} \exp \left(2 \kappa_{2} x\right), \quad x<0,
\end{array}\right.
$$

which expectedly presents the same result as Eq. (3.10) of Ref. [17], and

$$
\tilde{\mathcal{P}}_{M}^{\text {vol }}=g|A|^{2} \frac{k_{0}^{2}}{k_{p}} \overline{\mathbf{z}} \begin{cases}\exp \left(-2 \kappa_{1} x\right), & x>0 \\ \frac{1}{\varepsilon} \exp \left(2 \kappa_{2} x\right), & x<0 .\end{cases}
$$

Remarkably, the expression (38) coincides with the kinetic Abraham momentum $\mathcal{P}_{A}$ of the SPP obtained in Eq. (3.7) of Ref. [17] with no account of the dispersion:

$$
\mathcal{P}_{A}=\tilde{\mathcal{P}}_{M}^{\text {vol }} \text {. }
$$

This is an interesting conclusion. It suggests that the dispersion-modified kinetic Minkowski momentum can be equivalent to the dispersion-free Abraham momentum as an instrument for describing the energy flow and the group velocity [16, 17]. However, Eq. (39) is associated with a special form of the SPP field adopted in the present study and with a simple model of the metal permittivity given by Eqs. (21) and (22). It can hardly be generalized to other cases. More instructive and demonstrative are the singular terms in Eqs. (31), (33) and (36). In the next Section we consider their physical nature via the microscopic analysis.

\section{Microscopic approach to SPP momentum}

Here we briefly consider how the modifications of the SPP momentum description brought about by the new definitions of the field momentum in the dispersive medium (see Eqs. (20), (14a), (31), (36) and (38)) are compatible with the microscopic analysis. Following Refs. [16, 17], the microscopic approach is based on separation of the microscopic electromagnetic field ( $\mathbf{E}$ and $\mathbf{H}$ ) and charges/currents inside the medium. The metal is described by the Bloch hydrodynamic model for electron plasma, in which the electron density is characterized by the uniform 'background' density $n_{0}$ (see Eq. (22)) modified by small additive time-harmonic perturbation, $\operatorname{Re}[\tilde{n}(\mathbf{r}) \exp (-i \omega t)]$, and the local velocity of electrons is taken in the form $\operatorname{Re}[\tilde{\mathbf{v}}(\mathbf{r}) \exp (-i \omega t)]$. 
Then the free-space Maxwell equations with $\varepsilon=\mu=1$ and with the densities of charge $e \tilde{n}$ and current $e n_{0} \tilde{\mathbf{v}}$,

$$
\nabla \cdot \mathbf{H}=0, \quad \mathbf{H}=-\frac{i}{k_{0}} \nabla \times \mathbf{E}, \quad \nabla \cdot \mathbf{E}=4 \pi e \tilde{n}, \quad \mathbf{E}=\frac{i}{k_{0}} \nabla \times \mathbf{H}-i \frac{4 \pi e n_{0}}{\omega} \tilde{\mathbf{v}},
$$

yield the following for the medium $1(x<0)$ :

$$
\mathbf{E}=\frac{A}{\varepsilon}\left\{\left[-(1-\varepsilon) e^{\gamma x}+e^{\kappa_{2} x}\right] \overline{\mathbf{x}}+i\left[-(1-\varepsilon) \frac{k_{p}}{\gamma} e^{\gamma x}+\frac{\kappa_{2}}{k_{p}} e^{\kappa_{2} x}\right] \overline{\mathbf{z}}\right\} \exp \left(i k_{p} z\right), x<0,
$$

(the magnetic field is still described by Eq. (24)), and

$$
\begin{gathered}
\tilde{n}=\frac{A}{4 \pi e} \frac{\varepsilon-1}{\varepsilon}\left(\gamma-\frac{k_{p}^{2}}{\gamma}\right) e^{\gamma x} \exp \left(i k_{p} z\right), x<0, \\
\tilde{\mathbf{v}}=i \frac{A}{\varepsilon} \frac{e}{m \omega}\left[\left(-e^{\gamma x}+e^{\kappa_{2} x}\right) \overline{\mathbf{x}}+i\left(-\frac{k_{p}}{\gamma} e^{\gamma x}+\frac{\kappa_{2}}{k_{p}} e^{\kappa_{2} x}\right) \overline{\mathbf{z}}\right] \exp \left(i k_{p} z\right), x<0 .
\end{gathered}
$$

Here the relation $\gamma^{2}=k_{p}^{2}-\varepsilon \omega^{2} / \beta^{2}$ holds, where the coefficient $\beta^{2}=(3 / 5) v_{F}^{2}$ involving the Fermi velocity $v_{F}$ of electrons is responsible for the additional quantum pressure. The $\varepsilon$ parameter is still described by Eq. (21) although in Eqs. (41)-(43), like everywhere in this Section, it is not postulated but derived from the microscopic analysis. According to Refs. [16, 17], we imply the limit $\beta^{2} \rightarrow 0$ and, correspondingly, $\gamma \rightarrow \infty$ in our further consideration.

In the limit $\gamma \rightarrow \infty$, the $\gamma$-containing terms are nonzero only in the closest vicinity of the interface in the metal half-space $x<0$, and we will call them as 'near-surface terms'. Though their contributions seem to be negligible, we keep them explicitly because they are crucial to fulfil the boundary conditions (i.e., continuity of the electric field and a zero normal velocity of electrons at $x=0$ - see Eq. (43)) and additionally characterize the near-surface behaviour of the field characteristics. Besides, in some cases such terms provide specific non-vanishing nearsurface contributions due to the limiting transition

$$
\gamma \exp (\gamma x) \rightarrow \delta(x)
$$

Now let us consider the momentum calculation for the metal. According to Refs. [8-10], the electromagnetic momentum includes the field and material contributions, where the field contribution is described by the Poynting vector of the free-space field of Eqs. (40),

$$
\mathcal{P}_{0}=g k_{0} \operatorname{Re}\left(\mathbf{E}^{*} \times \mathbf{H}\right) \text {. }
$$

In view of Eqs. (40), its spin-orbital decomposition reads as (cf. with Eqs. (7)-(9))

$$
\begin{aligned}
\mathcal{P}_{0}= & \underbrace{\frac{g}{2} \operatorname{Im}\left[\mathbf{E}^{*} \cdot(\nabla) \mathbf{E}+\mathbf{H}^{*} \cdot(\nabla) \mathbf{H}\right]-2 \pi g \frac{n_{0} e}{c} \operatorname{Im}\left(\mathbf{H}^{*} \times \tilde{\mathbf{v}}\right)}_{\text {canonical }} \\
& +\underbrace{\frac{g}{4} \nabla \times \operatorname{Im}\left[\left(\mathbf{E}^{*} \times \mathbf{E}\right)+\left(\mathbf{H}^{*} \times \mathbf{H}\right)\right]-2 \pi g e \operatorname{Im}\left(\mathbf{E}^{*} \tilde{n}\right)}_{\text {spin }} .
\end{aligned}
$$

The material contribution is calculated considering a long but finite wave packet and a cycleaveraged force density acting on the dipole moment induced in the medium by an external electromagnetic field $[10,17]$. Afterwards, the length of the wave packet tends to infinity, with the 
result

$$
\mathcal{P}_{\text {mat }}=\frac{g \omega}{2} \frac{d \varepsilon}{d \omega} \operatorname{Im}\left[\mathbf{E}^{*} \cdot(\nabla) \mathbf{E}\right]+(\varepsilon-1) g k_{0} \operatorname{Re}\left(\mathbf{E}^{*} \times \mathbf{H}\right) .
$$

In combination with Eq. (45), this gives the kinetic momentum that corresponds to the Philbin's expression (14):

$$
\tilde{\mathcal{P}}_{M}^{P}=\varepsilon \underbrace{g k_{0} \operatorname{Re}\left(\mathbf{E}^{*} \times \mathbf{H}\right)}_{\mathcal{P}_{0}}+\frac{g \omega}{2} \frac{d \varepsilon}{d \omega} \operatorname{Im}\left[\mathbf{E}^{*} \cdot(\nabla) \mathbf{E}\right] .
$$

Applying this result to the SPP field (41), we get

$$
\tilde{\mathcal{P}}_{M}^{P}=g|A|^{2} \frac{k_{0}^{2}}{k_{p}} \overline{\mathbf{z}}\left[-\frac{1-\varepsilon}{\varepsilon(1+\varepsilon)} e^{\left(\kappa_{2}+\gamma\right) x}+\frac{1-\varepsilon+2 \varepsilon^{2}}{\varepsilon(1+\varepsilon)} e^{2 \kappa_{2} x}\right], \quad x<0,
$$

which, excluding the near-surface term, coincides with the phenomenological expression given by Eq. (37). The canonical and spin parts of this momentum follow from decomposition of the $\mathcal{P}_{0}$ term in Eq. (47) with using Eq. (46). Then we obtain, with the aid of Eqs. (A1) and (A2) (see Appendix),

$$
\tilde{\mathbf{P}}_{M}=g|A|^{2} \frac{k_{0}^{2}}{k_{p}} \overline{\mathbf{z}}\left[\frac{(1-\varepsilon)^{2}}{2(1+\varepsilon)} e^{2 \gamma x}-\frac{(1-\varepsilon)(2-\varepsilon)}{2 \varepsilon} e^{\left(\gamma+\kappa_{2}\right) x}+\frac{1-\varepsilon+\varepsilon^{2}}{\varepsilon(1+\varepsilon)} e^{2 \kappa_{2} x}\right], \quad x<0
$$

and

$$
\mathbf{P}_{M}^{S}=g|A|^{2} \frac{k_{0}^{2}}{k_{p}} \overline{\mathbf{z}}\left[\frac{(1-\varepsilon)^{2}}{2(1+\varepsilon)}\left(-e^{\gamma x}+e^{\kappa_{2} x}\right) e^{\gamma x}+\frac{\varepsilon}{1+\varepsilon} e^{2 \kappa_{2} x}\right], \quad x<0 .
$$

The both results correspond well to the macroscopic relations (26) and (32). Note that, due to the near-surface terms in Eq. (49), the microscopic canonical momentum is continuous at the interface $x=0$ (cf. with Eq. (26)).

To find further momentum constituents, let us address microscopically the spin AM of the SPP field. Since the spin contains only the $y$-component, Eq. (30) gives a direct way to the spin constituent associated with the spin momentum (50),

$$
\mathbf{S}_{M}=g|A|^{2} \frac{k_{0}^{2}}{k_{s}} \overline{\mathbf{y}} \frac{1}{\varepsilon+1}\left(-\frac{(1-\varepsilon)^{2}}{2 \gamma} e^{2 \gamma x}+\frac{(1-\varepsilon)^{2}}{\gamma+\kappa_{2}} e^{\left(\gamma+\kappa_{2}\right) x}+\frac{\varepsilon}{\kappa_{2}} e^{2 \kappa_{2} x}\right), x<0,
$$

which reduces to Eq. (29) upon the condition $\gamma \rightarrow \infty$. This is the 'naïve' Minkowski spin which, according to Eq. (4.21) of Ref. [17], equals to

$$
\mathbf{S}_{M}=\frac{g}{2} \operatorname{Im}\left(\varepsilon \mathbf{E}^{*} \times \mathbf{E}\right) .
$$

There exists another spin constituent associated with the elliptic motion of free electrons driven by the rotating electric field (23) or (41) (see Fig. 2). It has been considered in Ref. [17] and described by the relation

$$
\mathbf{S}_{\mathrm{mat}}=\frac{n_{0} m}{2 \omega} \operatorname{Im}\left(\tilde{\mathbf{v}}^{*} \times \tilde{\mathbf{v}}\right)=\frac{n_{0} e^{2}}{2 m \omega^{3}} \operatorname{Im}\left(\mathbf{E}^{*} \times \mathbf{E}\right)=\frac{g \omega}{2} \frac{d \varepsilon}{d \omega} \operatorname{Im}\left(\mathbf{E}^{*} \times \mathbf{E}\right) .
$$

This 'material' spin corresponds to the dispersion terms in Eq. (15), and the sum $\tilde{\mathbf{S}}_{M}=\mathbf{S}_{M}+\mathbf{S}_{\text {mat }}$ forms the true dispersion-modified spin (19) for the SPP. Therefore, to finalize the SPP 


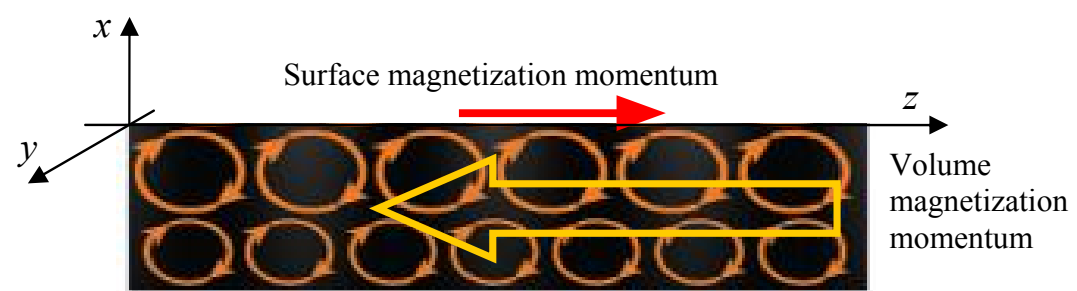

Fig. 2. Illustration of elliptic trajectories of electrons and magnetization momentum. The size of the ellipses and the electron velocities decay with increasing off-surface distance in the half-space $x<0$.

momentum calculation, we should determine the quantity whose macroscopic prototype is presented by Eq. (33):

$$
\Delta \mathcal{P}=\frac{1}{2} \nabla \times\left(\tilde{\mathbf{S}}_{M}-\mathbf{S}_{M}\right)=\frac{1}{2} \nabla \times \mathbf{S}_{\mathrm{mat}} .
$$

The evaluation becomes straightforward with the known distributions of the SPP field (41) and the electron velocity (43). However, the different expressions (53) are not fully equivalent; the first equality is more accurate because, in further transformations, simple proportionality between $\tilde{\mathbf{v}}$ and $\mathbf{E}$ is supposed, with discarding the near-surface terms [17]. Therefore we use the 'original' form

$$
\Delta \mathcal{P}=\frac{n_{0} m}{4 \omega} \operatorname{Im}\left[\nabla \times\left(\tilde{\mathbf{v}}^{*} \times \tilde{\mathbf{v}}\right)\right] .
$$

Based on Eq. (43), it can be transformed by the procedure described in Appendix. Eventually, it yields the following formulae for $x \leq 0$ :

$$
\begin{aligned}
\mathbf{S}_{\text {mat }} & =-g|A|^{2} \frac{2 \kappa_{2}}{k_{p}} \overline{\mathbf{y}} \frac{1-\varepsilon}{\varepsilon^{2}}\left[e^{2 \kappa_{2} x}-e^{\left(\kappa_{2}+\gamma\right) x}\right], \\
\Delta \mathcal{P} & =-g|A|^{2} \frac{1-\varepsilon}{\varepsilon^{2}} \overline{\mathbf{z}}\left[\frac{2 \kappa_{2}^{2}}{k_{p}} e^{2 \kappa_{2} x}-\frac{\kappa_{2}}{k_{p}} \delta(x)\right] \\
& =-g|A|^{2} \frac{k_{0}^{2}}{k_{p}} \overline{\mathbf{z}} \frac{2(1-\varepsilon)}{-1-\varepsilon} e^{2 \kappa_{2} x}+g|A|^{2} \frac{1-\varepsilon}{(-\varepsilon)^{3 / 2}} \overline{\mathbf{z}} \delta(x) .
\end{aligned}
$$

This is precisely the result given by Eqs. (33) and (34). Therefore, the microscopic model of the SPP confirms perfectly the results obtained in Section 3 uisng the general phenomenological approach.

As a final remark, we emphasize that the momentum $\Delta \mathcal{P}$ (more exactly, its volume part $\left.(\Delta \mathcal{P})^{\mathrm{vol}}\right)$ is in fact present in Refs. [16, 17], although not deduced from the general scheme of the momentum description in complex media. It appears in the analysis of metal magnetization effect due to rotational motion of free electrons (see subsection 4.4 of Ref. [17]), and is interpreted as a 'magnetization momentum' $\mathcal{P}_{\text {magn }}$. It is evident that its expression given by Eq. (4.29) of Ref.

[17] coincides completely with $(\Delta \mathcal{P})^{\text {vol }}$ (see Eq. (34)).

This means that due to the corrected expressions for the field momentum (16a) and (14a), the magnetization momentum finds its place in the unified picture of the field momentum and AM in complex media. Really, obeying the easily verifiable relation $\mathcal{P}_{\text {magn }}=\frac{1}{2} \nabla \times \mathbf{S}_{\text {mat }}$, it appears as a part of the linear spin momentum. Now, its immediate link with the vortex motion of electrons 
discloses a general physical mechanism of genesis of the spin momentum and deep analogies with the similar phenomena in, e.g., electromagnetism and fluid mechanics [29-34], where a linear macroscopic current emerges in the system of inhomogeneously distributed microscopic vorticities. In our case, the volume magnetization appears due to incomplete compensation of oppositely directed electron velocities in adjacent horizontal layers of the metal (see Fig. 2) and is proportional to the 'vorticity gradient' $\left(d S_{\text {mat }} / d x\right)$. Additionally, $S_{\text {mat }}$ abruptly changes to zero at the interface [This fact can be seen from the second term in parentheses of Eq. (55), which is zero almost everywhere in the volume at large $\gamma$ but rapidly increases to unity at $x=0$ ], which corresponds to the delta-like gradient though of the opposite sign. Therefore, the surface part in the expression (34), $(\Delta \mathcal{P})^{\text {surf }}$, should also be considered as a part of the magnetization momentum, in addition to the volume part discussed in Refs. [16, 17] (see Fig. 2). Accordingly, the true form of the magnetization momentum is $\mathcal{P}_{\text {magn }}=\Delta \mathcal{P}$ which appears in Eq. (34), with the singular part $\mathcal{P}_{\text {magn }}^{\text {surf }}=(\Delta \mathcal{P})^{\text {surf }}$.

This surface part of the magnetization momentum is similar to the surface Ampere current in magnets [32, 33]. It is noteworthy that, according to Eq. (35), the integral magnetization momentum over the whole SPP cross-section is $\left\langle\mathcal{P}_{\text {magn }}\right\rangle=\int_{-\infty}^{\infty} \Delta \mathcal{P} d x=0$, so that the surface (singular) current forms a 'closed circuit' with the volume (distributed) part.

\section{Conclusions}

The main result of this work is the unified description of the momentum and the AM in the lossless dispersive media, which is provided by Eq. (16a) and Eqs. (17)-(20). Due to enhanced interpretation of the known relation (13) and recognition that every sort of the spin AM is accompanied by the corresponding linear spin momentum, we have accomplished the recent scheme suggested in Refs. $[16,17]$ to its logical end. Now the system of equations for all the constituents (i.e., the canonical (orbital) and linear spin momenta, together with the orbital and spin AMs) appears in the perfect form, including neatly and concisely the dispersion corrections introduced in the same manner as in the well-known Brillouin formula (2), (3) for the energy. Importantly, all the other conclusions of Refs. $[16,17]$ remain unchanged. They are associated with physical interpretation of the kinetic and canonical pictures, meaningful discrepancies and appropriateness of the Abraham-type and Minkowski-type paradigms, as well as the novel effects predicted for the SPP physics.

Our refined general prescriptions have been applied to the SPP case which provides an example of highly structured field in a strongly inhomogeneous and essentially dispersive medium. The microscopic analysis supports completely the expectations based on the phenomenological grounds. Additionally, the magnetization momentum earlier introduced in Refs. [16, 17] upon considering the special effect of the SPP-induced magnetization, has now been included into the unified general scheme and appears to be its essential part. Moreover, its singular component associated with the surface current has been revealed and explained on the footing of far-reaching electromagnetic and hydrodynamic analogies.

Our microscopic analysis is based on the free-electron gas model for the metal but involves partly the quantum-pressure influence immanent in the hydrodynamic model of the electron plasma. Although this influence is supposed to be negligible, some residual effects have been taken into account in the form of corrections to the distributions of electric field, electron density 
and velocity, which are sharply localized near the metal-vacuum interface. These 'near-surface' terms do not affect the main volume properties of the SPP wave though provide conceptually meaningful contributions necessary for the boundary conditions to be fulfilled. Besides, they describe some principled details of the near-interface behaviour of various momentum components. In particular, it is these terms that stipulate the singular surface part of the magnetization momentum (34). Another remarkable observation is that the canonical momentum of the SPP field appears to be continuous at the interface (cf. Eqs. (49) and (26)). At the same time, the meaning and the consequences of the near-surface terms need additional elucidation and, probably, require a more accurate model for the electron properties of metals, which is a promising direction for further elaboration.

We hope that the present study provides a suitable and efficient toolkit for the analysis and description of the momentum and AM of light in dispersive and inhomogeneous (but isotropic and lossless) media. It can be used to solve a variety of modern problems, involving those related to photonic crystals, metamaterials and optomechanical systems.

\section{Appendix}

We consider transformations of the second line of Eq. (46). In the first term, the product $\mathbf{H}^{*} \times \mathbf{H}$ vanishes for the SPP field given by Eq. (24) and we must calculate the term $\frac{g}{4} \nabla \times \operatorname{Im}\left(\mathbf{E}^{*} \times \mathbf{E}\right)$ only. By substituting Eq. (41), we find

$$
\begin{aligned}
\frac{g}{4} \operatorname{Im}\left(\mathbf{E}^{*} \times \mathbf{E}\right) & =-\frac{g}{2} \overline{\mathbf{y}} \operatorname{Im}\left(E_{x}^{*} E_{z}\right) \\
& =-\frac{g|A|^{2}}{2 \varepsilon^{2}} \overline{\mathbf{y}}\left[(1-\varepsilon)^{2} \frac{k_{p}}{\gamma} e^{2 \gamma x}+\frac{\kappa_{2}}{k_{p}} e^{2 \kappa_{2} x}-(1-\varepsilon)\left(\frac{\kappa_{2}}{k_{p}}+\frac{k_{p}}{\gamma}\right) e^{\left(\kappa_{2}+\gamma\right) x}\right]
\end{aligned}
$$

Further, direct application of the rule (30) leads to representation

$$
\begin{array}{r}
\frac{g}{4} \operatorname{Im} \nabla \times\left(\mathbf{E}^{*} \times \mathbf{E}\right)=-\frac{g|A|^{2}}{2 \varepsilon^{2}} \overline{\mathbf{z}}\left[2 k_{p}(1-\varepsilon)^{2} e^{2 \gamma x}+\frac{2 \kappa_{2}^{2}}{k_{p}} e^{2 \kappa_{2} x}\right. \\
\left.-(1-\varepsilon)\left(\frac{\kappa_{2}}{k_{p}}+\frac{k_{p}}{\gamma}\right)\left(\kappa_{2}+\gamma\right) e^{\left(\kappa_{2}+\gamma\right) x}\right] \\
\underset{\gamma \rightarrow \infty}{\longrightarrow}-\frac{g|A|^{2}}{2 \varepsilon^{2}} \overline{\mathbf{z}}\left[2 k_{p}(1-\varepsilon)^{2} e^{2 \gamma x}-(1-\varepsilon)\left(\frac{\kappa_{2}^{2}}{k_{p}}+k_{p}\right) e^{\left(\kappa_{2}+\gamma\right) x}\right. \\
\left.+\frac{2 \kappa_{2}^{2}}{k_{p}} e^{2 \kappa_{2} x}-(1-\varepsilon) \frac{\kappa_{2}}{k_{p}} \delta(x)\right],
\end{array}
$$

where the limiting transition $\gamma \rightarrow \infty$ is performed and Eqs. (44) and (25) are employed.

In the second term of the second line of Eq. (46), substitution of Eqs. (41) and (42) accompanied with the same limiting transition and taking Eq. (44) into account gives

$$
\begin{aligned}
& -2 \pi g e \operatorname{Im}\left(\mathbf{E}^{*} \tilde{n}\right)=\frac{g}{2}|A|^{2} \frac{1-\varepsilon}{\varepsilon^{2}}\left[(1-\varepsilon) k_{p}\left(1-\frac{k_{p}^{2}}{\gamma^{2}}\right) e^{2 \gamma x}-\frac{\kappa_{2}}{k_{p}} \gamma e^{\kappa_{2} x+\gamma x}+\frac{\kappa_{2} k_{p}}{\gamma} e^{\kappa_{2} x+\gamma x}\right] . \\
& \underset{\gamma \rightarrow \infty}{\longrightarrow} \frac{g}{2}|A|^{2} \frac{1-\varepsilon}{\varepsilon^{2}}\left[(1-\varepsilon) k_{p} e^{2 \gamma x}-\frac{\kappa_{2}}{k_{p}} \delta(x)\right]
\end{aligned}
$$


Note that the terms with the delta functions in formulae (A1) and (A2) are mutually cancelled. Transformation of Eq. (54) is performed similarly to Eq. (A1), with taking into account Eq. (43), instead of Eq. (41).

\section{Acknowledgements.}

This work was supported by the Ministry of Education and Science of Ukraine (under the Project No. 582/18), RIKEN iTHES Project, MURI Center for Dynamic Magneto-Optics via the AFOSR Award No. FA9550-14-1-0040, the Japan Society for the Promotion of Science (KAKENHI), the IMPACT program of JST, the CREST grant No. JPMJCR1676, the John Templeton Foundation, the RIKEN-AIST “Challenge Research” program, and the Australian Research Council.

\section{References}

1. Halina Rubinsztein-Dunlop, Andrew Forbes, M V Berry, M R Dennis, David L Andrews, Masud Mansuripur, Cornelia Denz, Christina Alpmann, Peter Banzer, Thomas Bauer, Ebrahim Karimi, Lorenzo Marrucci, Miles Padgett, Monika Ritsch-Marte, Natalia M Litchinitser, Nicholas P Bigelow, C Rosales-Guzmán, A Belmonte, J P Torres, Tyler W Neely, Mark Baker, Reuven Gordon, Alexander B Stilgoe, Jacquiline Romero, Andrew G White, Robert Fickler, Alan E Willner, Guodong Xie, Benjamin McMorran and Andrew M Weiner, 2017. Roadmap on structured light. J. Opt. 19: 013001.

2. Andrews D L. Structured light and its applications: An introduction to phase-structured beams and nanoscale optical forces. : Academic Press (2011).

3. Bliokh K Y and Nori F, 2015. Transverse and longitudinal angular momenta of light. Phys. Rep. 592: 1-38.

4. Zayats A V, Smolyaninov I I and Maradudin A A, 2005. Nano-optics of surface plasmon polaritons. Phys. Rep. 408: 131-314.

5. Dienerowitz M, Mazilu M and Dholakia K, 2008. Optical manipulation of nanoparticles: a review. J. Nanophoton. 2: 021875.

6. Flores-Flores E, Torres-Hurtado S A, Páez R, Ruiz U, Beltrán-Pérez G, Neale S L, RamirezSan-Juan J C and Ramos-García R, 2015. Trapping and manipulation of microparticles using laser-induced convection currents and photophoresis. Biomed. Opt. Express. 6: 4079-4087.

7. Baffou G and Rigneault H, 2011. Femtosecond-pulsed optical heating of gold nanoparticles. Phys. Rev. B. 84: 035415.

8. Brevik I, 1979. Experiments in phenomenological electrodynamics and the electromagnetic energy-momentum tensor. Phys. Rep. 52: 133-201.

9. Barnett S M and Loudon R, 2010. The enigma of optical momentum in a medium. Phil. Trans. Roy. Soc. A. 368: 927-939.

10. Milonni P W and Boyd R W, 2010. Momentum of light in a dielectric medium. Adv. Opt. Photon. 2: 519-553.

11. Kemp B A, 2011. Resolution of the Abraham-Minkowski debate: Implications for the electromagnetic wave theory of light in matter. J. Appl. Phys. 109: 111101.

12. Landau L D, Lifshitz E M and Pitaevskii L P. Electrodynamics of continuous media. Oxford: Pergamon (1984).

13. Jackson J D. Classical electrodynamics ( $3^{\text {rd }}$ Ed). New York: Wiley (1999).

14. Philbin T G, 2011. Electromagnetic energy-momentum in dispersive media. Phys. Rev. A. 83: 013823; Erratum: 2012. Phys. Rev. A. 85 059902(E). 
15. Philbin T G and Allanson O, 2012. Optical angular momentum in dispersive media. Phys. Rev. A. 86: 055802.

16. Bliokh K Y, Bekshaev A Y and Nori F, 2017. Optical momentum, spin, and angular momentum in dispersive media. Phys. Rev. Lett. 119: 073901.

17. Bliokh K Y, Bekshaev A Y and Nori F, 2017. Optical momentum and angular momentum in dispersive media: From the Abraham-Minkowski debate to unusual properties of surface plasmon-polaritons. New J. Phys. 19: 123014.

18. Soper D E. Classical field theory. New York: Wiley (1976).

19. Yao A M and Padgett M J, 2011. Optical angular momentum: origins, behavior, and applications. Adv. Opt. Photon. 3: 161-204.

20. Bliokh K Y, Bekshaev A Y and Nori F, 2014. Extraordinary momentum and spin in evanescent waves. Nature Commun. 5: 3300.

21. Bliokh K Y, Bekshaev A Y, Kofman A G and Nori F, 2013. Photon trajectories, anomalous velocities, and weak measurements: a classical interpretation. New J. Phys. 15: 073022.

22. Bekshaev A Y, Bliokh K Y and Soskin M S, 2011. Internal flows and energy circulation in light beams. J. Opt. 13: 053001.

23. Bliokh K Y, Bekshaev A Y and Nori F, 2013. Dual electromagnetism: helicity, spin, momentum, and angular momentum. New J. Phys. 15: 033026; Corrigendum: 2016. New J. Phys. 18: 089503.

24. Leader E and Lorce C, 2014. The angular momentum controversy: What's it all about and does it matter? Phys. Rep. 541: 163-248.

25. Belinfante F J, 1940. On the current and the density of the electric charge, the energy, the linear momentum and the angular momentum of arbitrary fields. Physica. 7: 449-474.

26. Berry M V, 2009. Optical currents. J. Opt. A: Pure Appl. Opt. 11: 094001.

27. Bekshaev A Y, 2013. Subwavelength particles in an inhomogeneous light field: optical forces associated with the spin and orbital energy flows. J. Opt. 15: 044004.

28. Bliokh K Y and Nori F, 2012. Transverse spin of a surface polariton. Phys. Rev. A. 85: 061801(R).

29. Ohanian H C, 1986. What is spin? Amer. J. Phys. 54: 500-505.

30. Mita K, 2000. Virtual probability current associated with the spin. Amer. J. Phys. 68: 259264.

31. Witting C, 2009. Photon and electron spins. J. Phys. Chem. A. 113: 15320-15327.

32. Bekshaev A.Y, 2006. Spin angular momentum of inhomogeneous and transversely limited light beams. Proc. SPIE. 6254: 56-63.

33. Purcell E M. Electricity and magnetism (Berkeley Physics Course, Volume 2; $2^{\text {nd }}$ Ed.). : McGraw-Hill (1985).

34. Sedov L I. A course in continuum mechanics. Volume 3: Fluids, gases, and the generation of thrust. Groningen: Wolters-Noordhoff (1972).

Bekshaev A. Y. and Bliokh K. Y. 2018. Spin and momentum of the light fields in inhomogeneous dispersive media with application to surface plasmon-polariton waves. Ukr.J.Phys.Opt. 19: 33 - 48.

Анотація. На основі нещчодавно розробленого підходу [Phys. Rev. Lett. 119, 073901 (2017); New J. Phys., 19, 123014 (2017)] вдосконалено і завершено загальну схему уніфікованого опису імпульсу та кутового моменту в складних матеріальних середовищах. Рівняння для канонічного (орбітального) і спінового імпульсів, а також для орбітального та спінового 
кутових моментів у непоглинаючому неоднорідному диспергуючому середовищі представлено в компактній формі, аналогічній до формули Бриллюена для енергії. Результати застосовано до поля поверхневого плазмон-поляритона. Намі мікроскопічні розрахунки підтверджують очікування феноменології. Уточнена нами загальна схема правильно описує незвичні властивості поверхневого плазмон-поляритона, пов'язані 3 поперечним спіном та імпульсом намагнічування. На додаток, вона прогнозує сингулярні складові імпульсу, локалізовані на межі метал-діелектрик, щзо підтверджується мікроскопічним аналізом. Одержані результати можуть бути корисними для оптичних систем, що використовують структуроване світло, особливо в мікрооптиці, плазмофотоніці, оптичному сортуванні та мікроманіпуляціях. 